\title{
量子限域超流体技术用于氧化石墨烯智能化
}

\author{
张永来 ${ }^{1}$, 韩冬冬 ${ }^{1}$, 孙洪波 ${ }^{1,2^{*}}$
}

1. 吉林大学电子科学与工程学院, 集成光电子学国家重点实验室, 长春 130012;

2. 清华大学精密仪器系, 精密测试技术及仪器国家重点实验室, 北京 100084

* 联系人, E-mail: hbsun@ @tsinghua.edu.cn

\section{Quantum-confined-superfluidics-enabled moisture responsive graphene oxide papers}

\author{
Yong-Lai Zhang ${ }^{1}$, Dong-Dong Han $^{1} \&$ Hong-Bo Sun ${ }^{1,2^{*}}$ \\ ${ }^{1}$ State Key Laboratory of Integrated Optoelectronics, College of Electronic Science and Engineering, Jilin University, Changchun 130012, China; \\ ${ }^{2}$ State Key Laboratory of Precision Measurement Technology and Instruments, Department of Precision Instrument, Tsinghua University, Beijing 100084, \\ China \\ * Corresponding author, E-mail: hbsun@tsinghua.edu.cn \\ doi: 10.1360/TB-2020-0758
}

在生物系统中, 生物离子通道可以用于信息传输、能 量转换、质量转移. 例如: 人类通过生物离子通道感受多 种刺激(听觉、视觉、味觉等); 电鳗通过激活离子通道产生 超过 $600 \mathrm{~V}$ 的电压; 含羞草受到机械刺激时通过水在通道 内的快速流动引起叶柄下垂. 2018 年, 江雷教授团队 ${ }^{[1]}$ 将 离子和分子在限域孔道内有序快速传输的现象定义为“量 子限域超流体效应 (quantum confined superfluidics, QSF)”, 并指出基于 QSF 概念的材料在仿生学、信息科学、医学等 领域拥有广阔的应用前景.

氧化石墨烯 (graphene oxide, GO) 是石墨烯材料的一 种衍生物, 利用 GO 制备 QSF 概念的器件主要基于 GO 的 以下三个特点: (1) 水分子与 $\mathrm{GO}$ 表面的氧基团之间通过氢 键进行强相互作用, 有利于水分子的快速吸附 ${ }^{[2]}$. (2) 在相 邻的 GO 片层之间, 水分子具有超低的摩擦力和较大的滑 移长度, 导致水分子在相邻 $\mathrm{GO}$ 片层间的超快传输 ${ }^{[3]}$. (3) $\mathrm{GO}$ 薄膜具有纳米层状结构, 层间距通常在 $6 \sim 12 \AA$ 之间, 并且层间距离可以通过水分子在 $\mathrm{GO}$ 层间的吸附和脱附进 行有效调节 ${ }^{[4]}$. 然而, GO 的 QSF 效应一直没有用于刺激响 应型驱动器的研制.

理论上, GO 膜可以实现水分子的快速吸附和超快传 输, 通过耦合另一种对水分子不响应材料层就可以制备对 水分子敏感的湿度响应驱动器. 但双层/多层材料结构的 驱动器在频繁发生形变过程中, 具有异质材料间脱附等不 足, 这限制了驱动器的长期稳定工作. 基于此, 本课题组 ${ }^{[5]}$ 受量子限域超流体技术启发, 制备具有非对称 QSF 通道的 $\mathrm{GO}$ 薄膜, 即单侧具有微结构的 GO 薄膜. 由于非对称 $\mathrm{QSF}$
通道的存在, 该薄膜具有较好的湿度响应特点. 相关工作 发表在 Advanced Materials.

研究团队从第一性原理出发, 通过第一性原理计算水 分子与石墨烯 ( $\mathrm{sp}^{2}$ 区域)之间结合能约为 $0.04 \mathrm{eV} / \mathrm{H}_{2} \mathrm{O}$, 而 水分子与 $\mathrm{GO}$ 表面羟基、环氧基和边缘羧基的结合能分别 约为 $0.20 、 0.23$ 和 $0.36 \mathrm{eV} / \mathrm{H}_{2} \mathrm{O}$. 并通过 $\mathrm{X}$ 射线衍射技术、 原子力显微镜、拉曼光谱仪测试不同湿度条件下的数据, 推测 GO 与水分子相互作用过程中对相邻 GO 纳米片层间 滑移的影响.

利用软模板法制备具有单侧微结构的 GO 驱动器, 即 将 $\mathrm{GO}$ 水溶液滴在聚二甲基硅氧烷(PDMS)光栅条纹模板 上, 在空气中干燥, 然后将单侧具有微结构的 GO 薄膜从 PDMS 模板表面揭下来. 制备的 GO 驱动器具有湿度刺激 响应变形大 $\left(\sim 8.7 \mathrm{~cm}^{-1}\right)$ 、速度快 $\left(1.24 \mathrm{~cm}^{-1} \mathrm{~s}^{-1}\right)$ 等优点. 此外, $\mathrm{GO}$ 驱动器发生形变的方向可以通过 $\mathrm{GO}$ 光栅条纹的取向 来控制, 且可以发生螺旋弯曲和手性弯曲.

除了 PDMS 软模板转写的方法, 具有单侧微结构的 GO 驱动器还可以利用其他模板转写的方法. 例如: 我们 将 $\mathrm{GO}$ 溶液滴到金属网表面, 待 $\mathrm{GO}$ 溶液晾干后, GO 薄膜 揭下来也具有明显的湿度刺激弯曲形变的特点, 该方法可 以简单方便地制备大面积湿度响应 GO 驱动器. 最后, 因 为基于 QSF 效应的湿度响应 GO 驱动器具有较快的响应速 度, 研究团队将 GO 驱动器制成仿树叶形状, 该“树叶”可 以捕捉活漂虫(图 1).

综上, 本工作将第一性原理数值模拟与实验数据结合, 详细研究水分子与 $\mathrm{GO}$ 片层相互作用对 $\mathrm{GO}$ 薄膜性质的影 


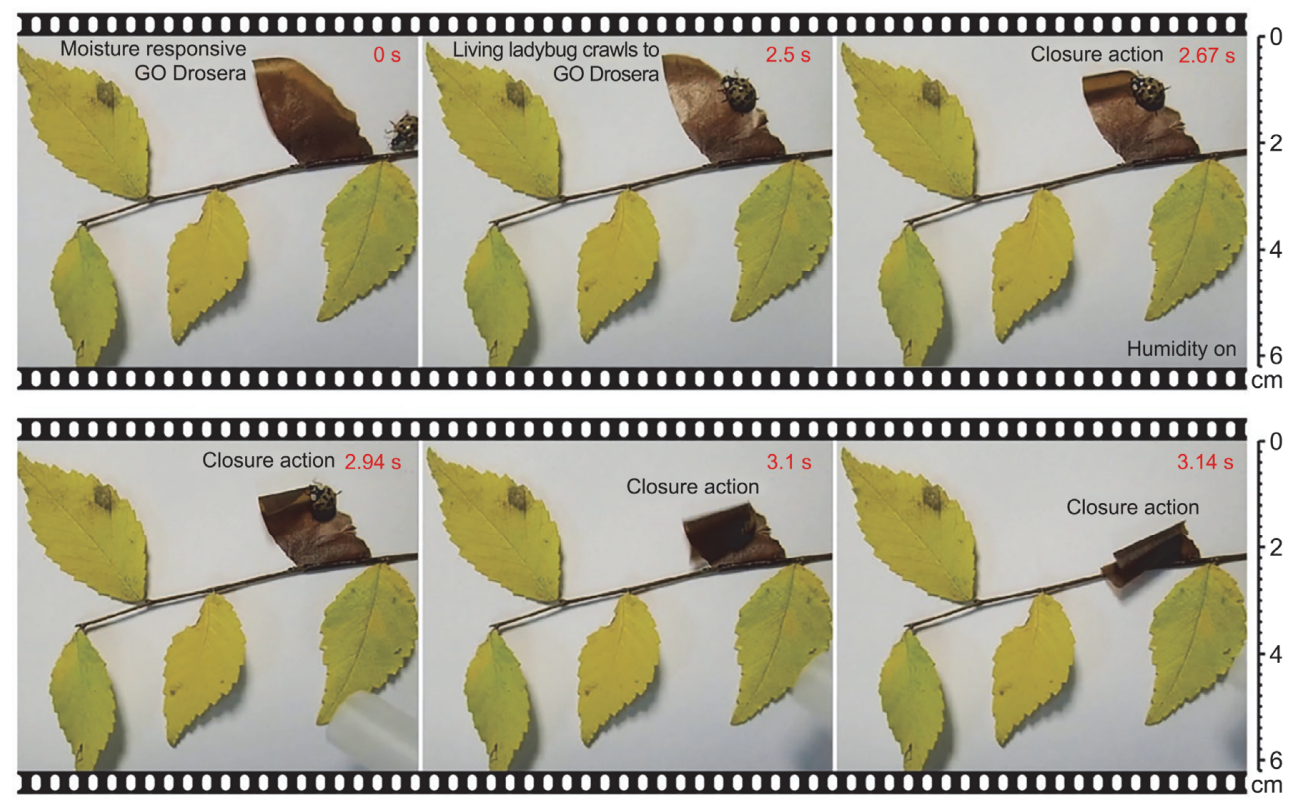

图 1 (网络版彩色)湿度响应氧化石墨烯膜用于捕捉瓢虫 ${ }^{[5]}$

Figure 1 (Color online) Moisture responsive graphene oxide papers catch the living ladybug ${ }^{[5]}$

响. 利用软模板转写法或金属网转写方法制备具有湿度响 应特性的单侧微结构的 GO 湿度响应驱动器, 该驱动器具 有响应变形大、速度快等优点. 此外, 该工作中所报道的
QSF 效应的驱动器设计理念还适用于 MXene 等其他二维 材料体系. 基于 QSF 通道的刺激响应驱动器有望在未来智 能微机器人的设计与开发中发挥重要作用.

\section{推荐阅读文献}

1 Zhang X Q, Liu H L, Jiang L. Wettability and applications of nanochannels. Adv Mater, 2019, 31: 1804508

2 Deng J, You Y, Bustamante H, et al. Mechanism of water transport in graphene oxide laminates. Chem Sci, 2017, 8: 1701-1704

3 Nair R R, Wu H A, Jayaram P N, et al. Unimpeded permeation of water through helium-leak-tight graphene-based membranes. Science, 2012, 335: 442-444

4 Abraham J, Vasu K S, Williams C D, et al. Tunable sieving of ions using graphene oxide membranes. Nat Nanotechnol, 2017, 12: 546

5 Zhang Y L, Liu Y Q, Han D D, et al. Quantum-confined-superfluidics-enabled moisture actuation based on unilaterally structured graphene oxide papers. Adv Mater, 2019, 31: 1901585 